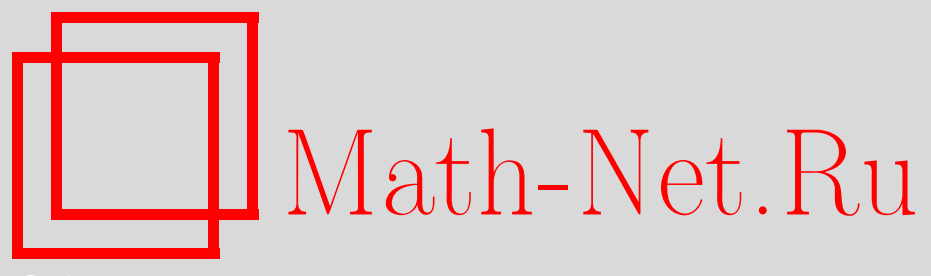

Х. Д. Икрамов, О приведении комплексных матриц к компактным формам посредством унитарных конгруэнций, Матем. заметки, 2007, том 82, выпуск 4, 550-559

DOI: https://doi.org/10.4213/mzm3803

Использование Общероссийского математического портала Math-Net.Ru подразумевает, что вы прочитали и согласны с пользовательским соглашением http://www . mathnet.ru/rus/agreement

Параметры загрузки:

IP : 52.205 .19 .152

26 апреля 2023 г., 13:38:24

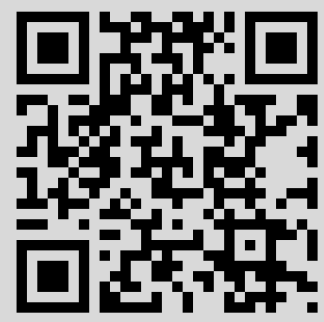


Том 82 выпуск 4 октябрь 2007

УДК 519.6

\section{О приведении комплексных матриц к компактным формам посредством унитарных конгруэнций}

\section{Х. Д. Икрамов}

Доказано, что всякая сопряженно-нормальная $(n \times n)$-матрица может быть посредством унитарной конгруэнции приведена к блочно-трехдиагональной форме, в которой порядки последовательных диагональных блоков не превосходят соответственно чисел $1,2,3, \ldots$. Доказательство конструктивное: описан конечный процесс, преобразующий матрицу к желаемой форме. Указаны достаточные условия для стабилизации порядков диагональных блоков. Компактная форма, соответствующая этому случаю, является ленточной матрицей.

Библиография: 3 названия.

1. Введение. Хорошо известно, что всякую эрмитову матрицу $A \in M_{n}(\mathbb{C})$ можно привести к трехдиагональному виду посредством конечной последовательности подобий с элементарными унитарными матрицами (отражениями или вращениями). Пусть $Q$ - произведение этих матриц и $T$ - полученная трехдиагональная форма. Тогда

$$
Q^{*} A Q=T .
$$

Обозначив столбцы матрицы $Q$ через $q_{1}, \ldots, q_{n}$ и переписав $(1)$ в виде

$$
A Q=Q T,
$$

получим

$$
\begin{aligned}
A q_{1}= & t_{11} q_{1}+t_{21} q_{2}, \\
A q_{2}= & t_{12} q_{1}+t_{22} q_{2}+t_{32} q_{3}, \\
& \ldots \ldots \ldots \ldots \\
A q_{n}= & t_{n-1, n} q_{n-1}+t_{n n} q_{n} .
\end{aligned}
$$

При этом

$$
\begin{aligned}
t_{i i} & =\left(A q_{i}, q_{i}\right), & & i=1,2, \ldots, n, \\
t_{21} & =\left\|A q_{1}-t_{11} q_{1}\right\|_{2}, & & \\
t_{i+1, i} & =\left\|A q_{i}-t_{i, i} q_{i}-t_{i-1, i} q_{i-1}\right\|_{2}, & & i=2,3, \ldots, n-1, \\
t_{i, i+1} & =t_{i+1, i}, & & i=1,2, \ldots, n-1 .
\end{aligned}
$$

Формулы (2)-(6) соответствуют еще одному хорошо известному методу унитарного приведения эрмитовой матрицы к трехдиагональному виду, называемому алгоритмом Ланиоша. Более привычное описание этого метода выглядит так: по 
заданному (нормированному) вектору $q_{1}$ строится степенная последовательность

$$
q_{1}, \quad A q_{1}, \quad A^{2} q_{1}, \quad \ldots,
$$

к которой применяется процесс ортогонализации, порождающий ортонормированные векторы Ланцоша $q_{1}, q_{2}, q_{3}, \ldots$. Первые $m$ векторов Ланцоша $(m=1,2, \ldots)$ составляют ортонормированный базис в $m$-м крыловском подпространстве

$$
\mathscr{K}_{m}=\mathscr{K}_{m}\left(A, q_{1}\right)=\operatorname{span}\left\{q_{1}, A q_{1}, \ldots, A^{m-1} q_{1}\right\} .
$$

Особенностью данного процесса по сравнению со стандартной ортогонализацией Грама-Шмидта является то, что на $m$-м шаге $(m>3)$ вектор $A q_{m-1}$ автоматически ортогонален подпространству $\mathscr{K}_{m-3}$. В результате вектор $q_{m}$ получается из ранее вычисленных векторов Ланцоша посредством трехчленной формулы типа (2), а не $m$-членного соотношения, как в общем случае. Этот результат, важный с вычислительной точки зрения, есть следствие трехдиагональности матрицы $T$ в (1).

Для матрицы $A$ общего вида процесс унитарного приведения дает вместо трехдиагональной (верхнюю) форму Хессенберга, т.е. матрицу $H$ такую, что $h_{i j}=0$ при $i>j+1$. Переходя от матричного равенства

$$
Q^{*} A Q=H
$$

к столбцевым соотношениям, получим формулы процесса, называемого в вычислительной линейной алгебре алгоритмом Арнольди.

И трехдиагональную форму, и форму Хессенберга принято называть компактными по той причине, что в обеих присутствует значительное количество нулевых элементов. Ясно, однако, что трехдиагональная матрица соответствует этому названию в гораздо большей мере. В связи с этим в литературе по линейной алгебре уже продолжительное время (с 1980-х годов) обсуждается следующий вопрос: какие матрицы (за пределами класса эрмитовых матриц) могут быть приведены (посредством конечной последовательности унитарных подобий) к компактным формам, по возможности мало отличающимся от трехдиагональной? Напомним сравнительно недавний результат в этом направлении [1], имеющий непосредственное отношение к тематике данной статьи.

Теорема 1. Пусть $A \in M_{n}(\mathbb{C})$ - нормальная матрица. Существует процесс типа Ланцоша, приводящий $А$ блочно-трехдиагональной форме

$$
H=\left(\begin{array}{cccc}
H_{11} & H_{12} & & \\
H_{21} & H_{22} & H_{23} & \\
& H_{32} & H_{33} & \ldots \\
& \ldots & \ldots & \ldots
\end{array}\right)
$$

где диагональные блоки $H_{11}, H_{22}, \ldots$ квадратные и их порядки в типичном случае даются последовательными натуральными числами $1,2,3, \ldots$ Если А удовлетворяет уравнению вида

$$
g\left(A, A^{*}\right)=0,
$$

где $g(x, y)$ - многочлен степени $k \ll n$, то порядки диагональных блоков $H_{i i}$ в матрище (8), начиная с $i=k$, стабилизируются на значении $k$. 
Более подробно процесс, приводящий к форме (8), обсуждается в п. 3. С полным основанием эту форму можно считать компактной, поскольку число ненулевых элементов в ней не превосходит $O\left(n^{3 / 2}\right)$ (тогда как в матрице Хессенберга примерно половина элементов отличны от нуля). Для матрицы $A$, удовлетворяющей уравнению (9), форму (8) можно рассматривать как ленточную матрицу, а число ненулевых элементов $h_{i j}$ есть линейная функция от $n$.

Если вместо унитарных подобий говорить об унитарных конгруэнциях, то известны следующие факты.

1. Всякую симметричную матрицу $A \in M_{n}(\mathbb{C})$ можно привести к трехдиагональному виду посредством конечной последовательности конгруэнций с элементарными унитарными матрицами. Результирующее преобразование описывается формулой

$$
Q^{T} A Q=T
$$

где $Q$ - унитарная матрица.

2. Аналогичный процесс элементарных унитарных конгруэнций приводит матрицу $A$ общего вида к форме Хессенберга.

Автору не известны какие-либо результаты о компактных формах, промежуточных между трехдиагональными и хессенберговыми матрицами.

Цель настоящей статьи - установить такой результат, взяв теорему 1 в качестве образца. При этом нужно рассматривать сопряженно-нормальные матрицы, играющие в теории унитарных конгруэнций ту же роль, какую обычные нормальные матрицы выполняют в теории унитарных подобий. Необходимые сведения о сопряженно-нормальных матрицах и доказательство главного результата даны в пп. 4 и 5. Основной технический прием, используемый в доказательстве, а именно, введение вспомогательной матрицы удвоенного порядка, обсуждается в п. 2.

2. Приведение к трехдиагональному виду. Пусть $A$ - комплексная симметричная матрица. Вернемся к равенству (10) и проинтерпретируем его в том же духе, в каком равенство (1) было интерпретировано во введении. Переписав (10) в виде

$$
A Q=\bar{Q} T
$$

получим для столбцов $q_{1}, \ldots, q_{n}$ матрицы $Q$ следующие соотношения:

$$
\begin{aligned}
A q_{1}= & t_{11} \bar{q}_{1}+t_{21} \bar{q}_{2}, \\
A q_{2}= & t_{12} \bar{q}_{1}+t_{22} \bar{q}_{2}+t_{32} \bar{q}_{3}, \\
& \ldots \ldots \ldots \ldots \\
A q_{n}= & t_{n-1, n} \bar{q}_{n-1}+t_{n n} \bar{q}_{n} .
\end{aligned}
$$

При этом

$$
\begin{aligned}
t_{i i} & =\left(A q_{i}, \bar{q}_{i}\right), & & i=1,2, \ldots, n, \\
\left|t_{21}\right| & =\left\|A q_{1}-t_{11} \bar{q}_{1}\right\|_{2}, & & \\
\left|t_{i+1, i}\right| & =\left\|A q_{i}-t_{i, i} \bar{q}_{i}-t_{i-1, i} \bar{q}_{i-1}\right\|_{2}, & & i=2,3, \ldots, n-1, \\
t_{i, i+1} & =t_{i+1, i}, & & i=1,2, \ldots, n-1 .
\end{aligned}
$$

Черта над символом матрицы или вектора обозначает поэлементное сопряжение. 
Формулы (11)-(15) соответствуют методу CSYM, предложенному в [2] для решения линейных систем с комплексными симметричными матрицами. По своему типу CSYM относится к методам минимальных невязок, однако отличается от других известных представителей этого класса (таких методов, как MINRES и GMRES) тем, что используемые в нем пробные подпространства не являются крыловскими. Более точная формулировка такова: положим

$$
\mathscr{N}_{m}=\operatorname{span}\left\{q_{1}, \ldots, q_{m}\right\}, \quad 1 \leqslant m \leqslant n .
$$

Тогда (см. [2; предложение 2])

$$
\mathscr{N}_{m}=\operatorname{span}\left\{q_{1}, \bar{A} \bar{q}_{1}, \bar{A} A q_{1},(\bar{A} A) \bar{A} \bar{q}_{1}, \ldots,(\bar{A} A)^{l-1} q_{1},(\bar{A} A)^{l-1} \bar{A}_{q_{1}}\right\},
$$

если $m=2 l$, и

$$
\mathscr{N}_{m}=\operatorname{span}\left\{q_{1}, \bar{A} \bar{q}_{1}, \bar{A} A q_{1}, \ldots,(\bar{A} A)^{l-1} \bar{A} \bar{q}_{1},(\bar{A} A)^{l} q_{1}\right\}
$$

если $m=2 l+1$. Таким образом, степенные последовательности, участвующие в формировании подпространств $\mathscr{N}_{m}$, порождаются матрицей

$$
A_{L}=\bar{A} A
$$

а не $A$. В отличие от обычных крыловских процессов, здесь две степенных последовательности вместо одной; их начальными векторами являются $q_{1}$ и $\bar{A} \bar{q}_{1}$.

Несмотря на сказанное, мы покажем, что CSYM может быть описан на языке крыловских подпространств. Точнее, CSYM может рассматриваться как проекция на $\mathbb{C}^{n}$ крыловского процесса, идущего в пространстве удвоенной размерности.

Сопоставим всякой матрице $A \in M_{n}(\mathbb{C})$ матрицу удвоенного порядка

$$
\widehat{A}=\left(\begin{array}{cc}
0 & \bar{A} \\
A & 0
\end{array}\right) \text {. }
$$

Известно (см. [3; теорема 2.1]), что спектр этой матрицы симметричен относительно нуля:

$$
\operatorname{Sp}(\widehat{A})=\left\{\mu_{1}, \ldots, \mu_{n},-\mu_{1}, \ldots,-\mu_{n}\right\} .
$$

Будем считать, что $\mu_{1}, \ldots, \mu_{n}$ принадлежат замкнутой правой полуплоскости. Тогда эти числа совпадают с псевдособственными значениями матрицы $A$, как они определены в [3], т.е. с квадратными корнями, имеющими неотрицательные вещественные части, из собственных значений матрицы $A_{L}$ (или, что все равно, матрицы $\left.A_{R}=A \bar{A}\right)$. Ввиду особенностей спектра $\operatorname{Sp} A_{L}$, невещественные псевдособственные значения в множестве $\left\{\mu_{1}, \ldots, \mu_{n}\right\}$ распадаются на сопряженные пары.

Легко видеть, что четные степени матрицы (20) имеют блочно-диагональный вид:

$$
\widehat{A}^{2 m}=A_{L}^{m} \oplus A_{R}^{m} .
$$

Нечетные степени имеют ту же структуру, что и сама матрица $\widehat{A}$ :

$$
\widehat{A}^{2 m+1}=\left(\begin{array}{cc}
0 & A_{L}^{m} \bar{A} \\
A_{R}^{m} A & 0
\end{array}\right) .
$$


Пусть теперь $A=A^{T}$. Очевидно, что матрица $\widehat{A}$ в этом случае является эрмитовой. Фиксируем нормированный вектор $q_{1} \in \mathbb{C}^{n}$ и положим

$$
v=\left(\begin{array}{c}
q_{1} \\
\bar{q}_{1}
\end{array}\right) \in \mathbb{C}^{2 n} .
$$

Степенная последовательность, порождаемая матрицей $\widehat{A}$ и вектором $v$, состоит из векторов

$$
v, \quad \widehat{A} v=\left(\begin{array}{l}
\bar{A} \bar{q}_{1} \\
A q_{1}
\end{array}\right), \quad \widehat{A}^{2} v=\left(\begin{array}{c}
A_{L} q_{1} \\
A_{R} \bar{q}_{1}
\end{array}\right), \quad \widehat{A}^{3} v=\left(\begin{array}{c}
A_{L} \bar{A} \bar{q}_{1} \\
A_{R} A q_{1}
\end{array}\right), \quad \ldots
$$

Верхние половины этих векторов образуют последовательность

$$
q_{1}, \quad \bar{A} \bar{q}_{1}, \quad A_{L} q_{1}, \quad A_{L} \bar{A} \bar{q}_{1}, \quad \ldots
$$

конечные сегменты которой определяют подпространства (17) и (18).

Если матрица $T$ в формуле (10) неприводима (т.е. все поддиагональные элементы $t_{i+1, i}$ отличны от нуля), то размерности подпространств $\mathscr{N}_{m}$ монотонно возрастают от 1 до $n$. Отсюда следует, что первые $n$ членов последовательности (25) линейно независимы.

Итак, для комплексной симметричной матрицы $A$ установлено соответствие между пробными подпространствами метода CSYM и крыловскими подпространствами вспомогательной матрицы $\widehat{A}$. Мы хотели бы распространить это соответствие на более широкий класс сопряженно-нормальных матриц. При таком распространении изменяется содержание понятия крыловского подпространства, о чем будет сказано в следующем пункте.

3. Обобщенный процесс Ланцоша. Пусть $A \in M_{n}(\mathbb{C})$ - нормальная матрица. Для произвольного (ненулевого) вектора $b \in \mathbb{C}^{n}$ последовательность

$$
b, \quad A b, \quad A^{*} b, \quad A^{2} b, \quad A A^{*} b, \quad A^{* 2} b, \quad A^{3} b, \quad \cdots
$$

будем называть обобщенной степенной последовательностью, порожденной парой $(A, b)$.

Удобно рассматривать последовательность (27) как состоящую из сегментов длины соответственно $1,2,3,4, \ldots$. Сегмент с номером $k$, называемый $k$-м слоем, можно описать как совокупность векторов вида $u=W_{k}\left(A, A^{*}\right) b$, где $W_{k}(s, t)$ пробегает множество одночленов степени $k$ от (коммутирующих) переменных $s$ и $t$. Символ $W_{0}(s, t)$ обозначает пустое слово, так что $W_{0}(s, t) b$ есть попросту вектор $b$.

Суть обобщенного процесса Ланцоша состоит в ортогонализации последовательности (27). Пусть $v_{1}, v_{2}, v_{3}, \ldots$ - получаемые при этом ненулевые ортогональные векторы. Положим

$$
\mathscr{K}_{m}=\operatorname{span}\left\{v_{1}, \ldots, v_{m}\right\} .
$$

Обозначим через $\operatorname{orth}_{\mathscr{K}} w$ ортогональную составляющую в разложении вектора $w$ относительно линейного подпространства $\mathscr{K}$.

Обобщенный процесс Ланцоша задается следующим предписанием.

1. Выбрать ненулевой вектор $b$ и положить $v_{1}=b$. 
2. Пусть уже построены ненулевые векторы $v_{1}, \ldots, v_{m}$, составляющие ортогональный базис линейной оболочки первых $k_{m}$ векторов $u_{1}, \ldots, u_{k_{m}}$ последовательности (27). Если перпендикуляр orth $\mathscr{K}_{m} u_{k_{m}+1}$ не равен нулю, принять его в качестве вектора $v_{m+1}$ и положить $k_{m+1}=k_{m}+1$. В противном случае построить вектор orth $\mathscr{K}_{m} u_{k_{m}+2}$. Если он ненулевой, принять его в качестве $v_{m+1}$ и положить $k_{m+1}=k_{m}+2$ и т.д.

ЗАмЕчАНИЕ. Если все векторы $k$-го слоя последовательности (27) дают нулевые перпендикуляры на текущее подпространство $\mathscr{K}_{m}$, то $\mathscr{K}_{m}$ есть инвариантное подпространство матрицы $A$ (следовательно, и матрицы $\left.A^{*}\right)$. Процесс Ланцоша в этом случае завершается досрочно.

В дальнейшем мы пользуемся следующей терминологией. Обобщенным nодnространством Крылова (с номером $m$ ) называется подпространство

$$
\mathscr{L}_{m}(A, b)=\operatorname{span}\left\{W\left(A, A^{*}\right) b: \operatorname{deg} W \leqslant m\right\} .
$$

Его размерность обозначается через $\ell_{m}$. Число $\omega_{m}=\ell_{m}-\ell_{m-1}, m \geqslant 1$, называется шириной $m$-го слоя; мы полагаем $\omega_{0}=1$. Иначе $\omega_{m}$ можно определить как размерность фактор-пространства $\mathscr{L}_{m} / \mathscr{L}_{m-1}$.

Предположим, что векторы $v_{1}, v_{2}, \ldots$, построенные в процессе Ланцоша, образуют базис пространства $\mathbb{C}^{n}$ (т.е. процесс не завершился досрочно). Нормируем их и полученные ортонормированные векторы $q_{1}, \ldots, q_{n}$ будем называть векторами Ланцоша. По построению, $q_{1}$ есть базисный вектор подпространства $\mathscr{L}_{0}$; векторы $q_{1}, q_{2}, q_{3}$ составляют (ортонормированный) базис подпространства $\mathscr{L}_{1}$; вообще, первые $\ell_{m}, m \geqslant 1$, векторов Ланцоша являются (ортонормированным) базисом подпространства $\mathscr{L}_{m}$. В этих терминах $\omega_{m}$ есть число векторов Ланцоша, добавленных к базису подпространства $\mathscr{L}_{m-1}$ посредством ортогонализации векторов в $m$-м слое последовательности (27). Естественно разбить на слои и сам базис Ланцоша, считая вектор $q_{1}$ его нулевым слоем, векторы $q_{2}$ и $q_{3}$ - первым слоем, и т.д.

Покажем, что обобщенный процесс Ланцоша можно рассматривать как метод унитарного приведения матрицы $A$ к блочно-трехдиагональной форме (8). Будем интерпретировать $A$ как линейный оператор, действующий в $\mathbb{C}^{n}$, и составим матрицу этого оператора в базисе из векторов Ланцоша. Примем во внимание следующие свойства обобщенных крыловских подпространств (см. [1; раздел 2]):

1) если $x \in \mathscr{L}_{m}$, то

$$
A x \in \mathscr{L}_{m+1}, \quad A^{*} x \in \mathscr{L}_{m+1}
$$

2) если $q_{\ell} \in \mathscr{L}_{m} \backslash \mathscr{L}_{m-1}$, то

$$
A q_{\ell} \perp \mathscr{L}_{m-2}, \quad A^{*} q_{\ell} \perp \mathscr{L}_{m-2} .
$$

В применении к векторам Ланцоша свойство 1) означает, что векторы вида $A q$, где $q$ пробегает $m$-й слой базиса Ланцоша, имеют нулевые коэффициенты Фурье по векторам из слоев с номерами, бо́льшими $m+1$. Из свойства 2) следует, что те же векторы $A q$ имеют нулевые коэффициенты и по векторам из слоев $0,1, \ldots, m-2$. Таким образом, в $(m+1)$-м блочном столбце матрицы $H$ отличны от нуля только блоки $H_{m, m+1}, H_{m+1, m+1}$ и $H_{m+2, m+1}, m=0,1,2, \ldots$, т.е. $H$ является блочно-трехдиагональной матрицей. Унитарная матрица $Q$, составленная из векторов $q_{1}, \ldots, q_{n}$, осуществляет приведение $A$ к форме $H$. 
4. Сопряженно-нормальные матрицы. Матрица $A \in M_{n}(\mathbb{C})$ называется сопряженно-нормальной, если

$$
A A^{*}=\overline{A^{*} A} \text {. }
$$

Сопряженно-нормальными являются, в частности, симметричные и кососимметричные матрицы (подобно тому, как эрмитовы и косоэрмитовы матрицы входят в класс обычных нормальных матриц).

Легко проверить, что матрица $A \in M_{n}(\mathbb{C})$ тогда и только тогда будет сопряженно-нормальной, когда соответствующая ей матрица $\widehat{A}$ (см. (20)) нормальна в обычном смысле.

По аналогии с п. 2 фиксируем нормированный вектор $q_{1} \in \mathbb{C}^{n}$ и определим вектор $v$ формулой (24). Построим обобщенную степенную последовательность, порождаемую парой $\widehat{A}, v$. Учтем при этом, что

$$
\widehat{A}^{*}=\left(\begin{array}{cc}
0 & A^{*} \\
A^{T} & 0
\end{array}\right)
$$

и степени этой матрицы выражаются формулами, аналогичными (22) и (23). В результате получим последовательность

$$
\begin{array}{rlrl}
v, \quad \widehat{A} v=\left(\begin{array}{l}
\bar{A} \bar{q}_{1} \\
A q_{1}
\end{array}\right), & \widehat{A}^{*} v=\left(\begin{array}{ll}
A^{*} \bar{q}_{1} \\
A^{T} q_{1}
\end{array}\right), & \widehat{A}^{2} v=\left(\begin{array}{l}
A_{L} q_{1} \\
A_{R} \bar{q}_{1}
\end{array}\right), \\
\widehat{A} \widehat{A}^{*} v & =\left(\begin{array}{l}
\bar{A} A^{T} q_{1} \\
A A^{*} \bar{q}_{1}
\end{array}\right), \quad \widehat{A}^{* 2} v=\left(\begin{array}{l}
A^{*} A^{T} q_{1} \\
A^{T} A^{*} \bar{q}_{1}
\end{array}\right), \quad \ldots
\end{array}
$$

Верхние половины этих векторов образуют последовательность

$$
q_{1}, \quad \bar{A} \bar{q}_{1}, \quad A^{*} \bar{q}_{1}, \quad \bar{A} A q_{1}, \quad \bar{A} A^{T} q_{1}, \quad A^{*} A^{T} q_{1}, \quad \bar{A} A \bar{A} \bar{q}_{1}, \quad \ldots
$$

Проведем ортогонализацию последовательности (32). Вектор $q_{1}$ уже нормирован. На втором шаге ортогонализуем вектор $A q_{1}$ к $\bar{q}_{1}$ :

$$
w_{2}=A q_{1}-h_{11} \bar{q}_{1}, \quad h_{11}=\left(A q_{1}, \bar{q}_{1}\right) .
$$

Теперь полагаем

$$
h_{21}=\left\|w_{2}\right\|_{2}, \quad q_{2}=\frac{\bar{w}_{2}}{h_{21}} .
$$

Эти соотношения означают, что $q_{2} \perp q_{1}$ и

$$
q_{2} \in \operatorname{span}\left\{q_{1}, \bar{A} \bar{q}_{1}\right\}
$$

как и должно быть.

На третьем шаге ортогонализуем $A^{T} q_{1}$ к $\bar{q}_{1}$ и $\bar{q}_{2}$ :

$$
w_{3}=A^{T} q_{1}-h_{11} \bar{q}_{1}-h_{12} \bar{q}_{2} .
$$

Коэффициент $h_{11}$ имеет значение, указанное в (33), а

$$
h_{12}=\left(A^{T} q_{1}, \bar{q}_{2}\right)=\left(A q_{2}, \bar{q}_{1}\right) .
$$


Вычислив $w_{3}$, полагаем

$$
h_{13}=\left\|w_{3}\right\|_{2}, \quad q_{3}=\frac{\bar{w}_{3}}{h_{13}} .
$$

Из (35) и (36) выводим, что

$$
q_{3} \in \operatorname{span}\left\{q_{1}, q_{2}, A^{*} \bar{q}_{1}\right\}
$$

Очевидно, что это включение согласуется с первыми тремя членами последовательности (32) (см. (34)).

В соответствии с соглашением, принятым в п. 3, последовательность (31) разбита на слои. Установим аналогичное разбиение для последовательности (32). Таким образом, векторы $\bar{A} \bar{q}_{1}$ и $A^{*} \bar{q}_{1}$, (неявно) участвовавшие во втором и третьем шагах ортогонализации, составляют первый слой, а $q_{2}$ и $q_{3}$ составляют первый слой будущего ортонормированного базиса $q_{1}, \ldots, q_{n}$.

Следующие три шага процесса соответствуют ортогонализации векторов во втором слое последовательности (32). Практически она выполняется так: векторы $A q_{2}$, $A q_{3}$ и $A^{T} q_{2}$ последовательно ортогонализуются к уже вычисленным векторам $\bar{q}_{i}$, нормируются и превращаются соответственно в $q_{4}, q_{5}$ и $q_{6}$.

Смысл коэффициентов ортогонализации $h_{11}, h_{12}, \ldots$ и нормировочных коэффициентов $h_{21}, h_{13}, \ldots$ выявляется из равенств $(33),(35)$ и аналогичных равенств последующих шагов (ср. с (11)): эти коэффициенты образуют матрицу $H$, к которой $A$ приводится посредством унитарной конгруэнции с трансформирующей матрицей

$$
Q=\left(q_{1} \ldots q_{n}\right)
$$

Матрица $H$ имеет блочное устройство, показанное формулой (8), причем размеры блоков определяются шириной слоев в последовательности (32). Это утверждение обосновывается примерно так же, как для обобщенного процесса Ланцоша, и опирается на аналоги свойств 1) и 2), отмеченных в п. 3 . Пусть $\mathscr{L}_{m}=\mathscr{L}_{m}(\widehat{A}, v)$, $m=1,2, \ldots,-$ обобщенные крыловские подпространства, определяемые последовательностью (31), а $\mathscr{M}_{m}$ - их проекции на $\mathbb{C}^{n}$, определяемые последовательностью (32). Положим

$$
\mathscr{P}_{m}=\left\{x \in \mathbb{C}^{n} \mid \bar{x} \in \mathscr{M}_{m}\right\} .
$$

Обсуждение процесса ортогонализации, проведенное выше, показывает, что

$$
A q \in \mathscr{P}_{m+1} \quad \text { и } \quad A^{T} q \in \mathscr{P}_{m+1},
$$

если $q \in \mathscr{M}_{m}$, что соответствует свойству 1) из п. 3.

Пусть $q_{l} \in \mathscr{M}_{m} \backslash \mathscr{M}_{m-1}, m \geqslant 2$, а $q_{i} \in \mathscr{M}_{m-2}$. Тогда

$$
\left(A q_{l}, \bar{q}_{i}\right)=\left(q_{l}, A^{*} \bar{q}_{i}\right)=\left(A^{T} q_{i}, \bar{q}_{l}\right)=0,
$$

поскольку

$$
A^{T} q_{i} \in \mathscr{P}_{i+1} \subset \mathscr{P}_{m-1} \quad \text { и } \quad \bar{q}_{l} \perp \mathscr{P}_{m-1} .
$$

Аналогичной выкладкой показываем, что

$$
\left(A^{T} q_{l}, \bar{q}_{i}\right)=0
$$

Равенства (38) и (39) соответствуют свойству 2) из п. 3. 
5. Приведение к ленточной форме. Всякая линейная зависимость между членами последовательности (31) влечет за собой линейную зависимость между соответствующими членами последовательности (32). Отсюда следует, что ширина произвольного слоя в (32) не превосходит ширины одноименного слоя в (31).

Обратимся теперь к теореме 1 в той части, которая касается уравнения (9). Предположим, что для матрицы (20) соотношение

$$
g\left(\widehat{A}, \widehat{A}^{*}\right)=0
$$

выполнено при некотором $k \ll n$. В этом случае ширина любого слоя в (31) не превосходит $k$, каков бы ни был вектор $v$. Это же верно для слоев последовательности (32). Поэтому процесс ортогонализации из п. 4 приводит $A$ к блочно-трехдиагональной форме $H$, в которой максимальный порядок диагонального блока не превосходит $k$. Теперь матрицу $H$ можно рассматривать как ленточную с шириной ленты не большей, чем $3 k$.

Учитывая блочную структуру матриц $\widehat{A}$ и $\widehat{A}^{*}$, получаем, что уравнение (40) равносильно двум соотношениям вида

$$
\varphi\left(A, \bar{A}, A^{T}, A^{*}\right)=0, \quad \psi\left(A, \bar{A}, A^{T}, A^{*}\right)=0 .
$$

Ниже мы указываем функции $\varphi$ и $\psi$ для некоторых конкретных значений $k$. Сейчас же отметим, что рассуждения этого и предыдущего пунктов в совокупности эквивалентны доказательству следующей теоремы.

Теорема 2. Пусть $A \in M_{n}(\mathbb{C})$ - сопряженно-нормальная матрица. Тогда посредством унитарных конгруэнций $A$ может быть приведена к блочно-трехдиагональной форме (8), где диагоналъные блоки $H_{11}, H_{22}, \ldots$ квадратные и их порядки в типичном случае даются последовательными натуральными числами $1,2,3, \ldots$. Если А удовлетворяет при некотором $k$ системе уравнений (41), то порядки диагональных блоков в матрице (8) не превосходят $k$.

Эта теорема является аналогом теоремы 1 с унитарными конгруэнциями, заменяющими унитарные подобия.

Проиллюстрируем теорему 2 в части, касающейся системы (41), для наиболее важных частных случаев $k=1$ и $k=2$. Заметим, что уравнение (40), приведшее к (41), влечет за собой равенство

$$
g(\lambda, \bar{\lambda})=0
$$

для всякого $\lambda \in \operatorname{Sp}(\widehat{A})$. С учетом формулы (21) это означает, что псевдособственные значения $\mu_{1}, \ldots, \mu_{n}$ матрицы $A$ должны удовлетворять условиям

$$
\widetilde{\varphi}(\mu, \bar{\mu})=0, \quad \widetilde{\psi}(\mu, \bar{\mu})=0,
$$

вытекающим из (41).

При $k=1$ матрица $\widehat{A}$ должна подчиняться соотношению

$$
\alpha \widehat{A}+\beta \widehat{A}^{*}+\gamma I_{2 n}=0
$$

для некоторых комплексных чисел $\alpha, \beta$ и $\gamma$, причем $\alpha$ и $\beta$ должны быть отличны от нуля. Система (41) для этого случая имеет вид

$$
\gamma I_{n}=0, \quad \alpha A+\beta A^{T}=0,
$$


откуда

$$
\gamma=0, \quad|\alpha|=|\beta| .
$$

Нетрудно проверить, что для ненулевой матрицы $A$ равенство

$$
A^{T}=e^{i \varphi} A, \quad \varphi \in \mathbb{R},
$$

возможно лишь в двух случаях: $A=A^{T}$ и $A=-A^{T}$. Итак, соотношение

$$
f\left(\widehat{A}, \widehat{A}^{*}\right)=0
$$

где $f$ - линейный многочлен, может описывать лишь (комплексные) симметричные и кососимметричные матрицы $A$.

При $k=2$ матрица $\widehat{A}$ подчиняется соотношению

$$
c_{11} \widehat{A}^{2}+2 c_{12} \widehat{A} \widehat{A}^{*}+c_{22} \widehat{A}^{* 2}+2 c_{10} \widehat{A}+2 c_{20} \widehat{A}^{*}+c_{00} I_{2 n}=0,
$$

где хотя бы один из коэффициентов $c_{11}, c_{12}$ и $c_{22}$ не равен нулю. Соответствующая система (41) имеет вид

$$
\begin{aligned}
c_{11} A \bar{A}+2 c_{12} A A^{*}+c_{22} A^{T} A^{*}+c_{00} I_{n} & =0, \\
2 c_{10} A+2 c_{20} A^{T} & =0 .
\end{aligned}
$$

Как было показано выше, равенство (43) при ненулевых $c_{10}$ и $c_{20}$ означает, что $A$ симметрична или кососимметрична. В этом случае уравнение (42) накладывает дополнительные ограничения на ее псевдоспектр.

Если $c_{10}=c_{20}=0$, то $A$ есть сопряженно-нормальная матрица, псевдособственные значения которой удовлетворяют уравнению

$$
c_{11} \mu^{2}+2 c_{12}|\mu|^{2}+c_{22} \bar{\mu}^{2}+c_{00}=0 .
$$

В типичном случае это уравнение описывает центральную кривую второго порядка.

\section{СПИСОК ЦИТИРОВАННОЙ ЛИТЕРАТУРЫ}

[1] L. Elsner, Kh. D. Ikramov, "On a condensed form for normal matrices under finite sequences of elementary unitary similarities", Linear Algebra Appl., 254:1-3 (1997), 79-98.

[2] A. Bunse-Gerstner, R. Stöver, "On a conjugate gradient-type method for solving complex symmetric linear systems", Linear Algebra Appl., 287:1-3 (1999), 105-123.

[3] A. George, Kh. D. Ikramov, E. V. Matushkina, W.-P. Tang, "On a QR-like algorithm for some structured eigenvalue problems", SIAM J. Matrix Anal. Appl., 16:4 (1995), $1107-1126$.

\section{Х. Д. Икрамов}

Поступило

Московский государственный университет

12.07.2006

им. М. В. Ломоносова

E-mail: ikramov@cs.msu.su 